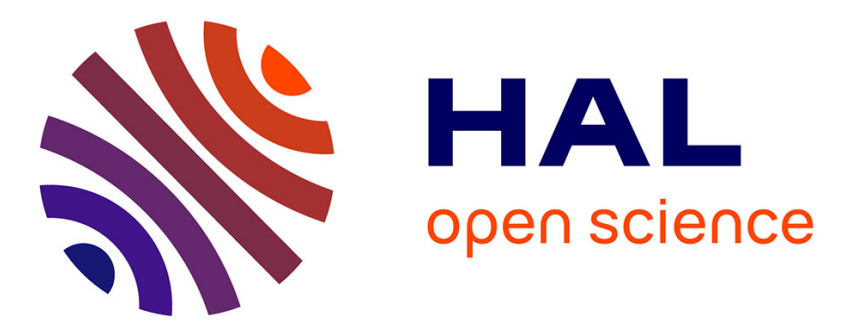

\title{
Secondary Instabilities and Vortex Formation in Collisionless-Fluid Magnetic Reconnection
}

\author{
Daniele del Sarto, Francesco Califano, Francesco Pegoraro
}

\section{To cite this version:}

Daniele del Sarto, Francesco Califano, Francesco Pegoraro. Secondary Instabilities and Vortex Formation in Collisionless-Fluid Magnetic Reconnection. Physical Review Letters, 2003, 91 (23), pp.235001. 10.1103/PhysRevLett.91.235001 . hal-01796224

\section{HAL Id: hal-01796224 \\ https://hal.science/hal-01796224}

Submitted on 19 May 2018

HAL is a multi-disciplinary open access archive for the deposit and dissemination of scientific research documents, whether they are published or not. The documents may come from teaching and research institutions in France or abroad, or from public or private research centers.
L'archive ouverte pluridisciplinaire HAL, est destinée au dépôt et à la diffusion de documents scientifiques de niveau recherche, publiés ou non, émanant des établissements d'enseignement et de recherche français ou étrangers, des laboratoires publics ou privés. 


\title{
Secondary Instabilities and Vortex Formation in Collisionless-Fluid Magnetic Reconnection
}

\author{
D. Del Sarto, F. Califano, and F. Pegoraro \\ Physics Department, University of Pisa and INFM, Pisa, Italy
}

(Received 29 May 2003; published 2 December 2003)

\begin{abstract}
It is shown that the pattern of current layers formed within a magnetic island in the nonlinear phase of magnetic field line reconnection in a collisionless two-dimensional fluid plasma is subject to the onset of a secondary instability, the effect of which increases with decreasing electron temperature. In the cold electron limit the saturation of the island growth is accompanied by a turbulent redistribution of the current layers and by the development of long lived fluid vortices while, in the opposite limit, the current layer structure remains regular.
\end{abstract}

DOI: 10.1103/PhysRevLett.91.235001

PACS numbers: 52.35.Vd, 52.30.Cv, 52.30.Ex, 52.35.We

The formation of current layers represents a generic feature of magnetic field line reconnection in high conductivity plasmas (see, e.g., [1] and references therein). It occurs in different reconnection regimes which include the Alfvénic frequency range of magnetohydrodynamics (MHD) and the higher frequency, whistler range, of electron magnetohydrodynamics (EMHD). These current structures arise as a consequence of the local magnetic linking (connections) between "fluid" plasma elements. Magnetic linking involves plasma fluid elements in the MHD frequency range, or electron fluid elements in the EMHD range, and is preserved when an (isotropic) fluid description of the plasma is applicable in the absence of phenomena occurring on scales fast in time and/or short in space. The breaking of the topological constraints due to magnetic linking, together with the process of magnetic energy release and transformation, determines the nonlinear evolution of the magnetic field configuration which leads to the formation of small scale spatial structures [2-4]. The role of magnetic topology is most important in dissipationless plasma regimes where the localized breaking of the magnetic connections is made possible not by the effect of electron resistivity but by the current limitation due to the finite electron inertia terms in the generalized Ohm's law. In these regimes the plasma behavior is Hamiltonian and "generalized" linking conditions [5] are preserved by its motions even in the presence of magnetic field line reconnection.

In this Letter we consider two-dimensional (2D), twofluid plasma regimes, where phase-space effects are disregarded and the plasma quantities can vary only in the plane perpendicular to a strong, externally imposed, uniform magnetic field component, and we focus our attention on the relationship between the generalized linking conditions and the time evolution of the current layers formed during the nonlinear development of the reconnection instability. By referring to both the MHD [2-5] and to the EMHD frequency ranges [6,7], we show that the long time evolution of these current layers can be strongly affected by the onset of a secondary Kelvin Helmholtz (K-H) instability which leads to the formation of fluid vortices inside the magnetic islands. Focusing on the MHD frequency range, we show that this behavior, which complements the "laminar" one described for a finite electron temperature plasma in Ref. [3], occurs in the cold electron regime where the effect of the electron parallel compressibility can be neglected. In addition, we show that this transition from the $\mathrm{K}-\mathrm{H}$ unstable regime to the laminar regime is related to the different nonlinear patterns that the current layers form because of the different magnetic linking conditions. The role of the K-H instability in the EMHD regime has been studied in the fully developed turbulence framework in Ref. [8].

We write the 2D magnetic field configuration in terms of the $z$ components of the vector potential $\psi(x, y, t)$ and of the magnetic field $b(x, y, t)$, as $\mathbf{B}=\mathbf{b}_{\perp}+b \mathbf{e}_{z}=\nabla \psi \times$ $\mathbf{e}_{z}+b \mathbf{e}_{z}$, while the ion velocity $\mathbf{u}$ in the $x-y$ plane is given in terms of the stream function $\varphi(x, y, t)$ as $\mathbf{u}=\mathbf{e}_{z} \times \nabla \varphi$. In the absence of dissipation and neglecting the Hall terms (i.e., neglecting perturbations of the large guide field $b$ ) the two-fluid equations in the MHD frequency range give [5]

$$
\begin{aligned}
\partial_{t} F+[\varphi, F] & =\varrho_{s}^{2}[U, \psi], \\
\partial_{t} U+[\varphi, U] & =[F, \psi] / d_{e}^{2},
\end{aligned}
$$

with $F=\psi-d_{e}^{2} \nabla^{2} \psi, U=\nabla^{2} \varphi,[f, g]=\mathbf{e}_{z} \cdot \nabla f \times \nabla g$. The effects of the electron inertia and of the parallel electron compressibility (proportional to the electron temperature) are included in Eqs. (1) via the the collisionless electron skin depth $d_{e}$ and the so-called ion-sound gyroradius $\varrho_{s}$, respectively. In the EMHD frequency range, the ions are assumed to be immobile (i.e., $\varphi=0$ ) and, neglecting electron temperature and charge separation effects, the cold fluid electron equations give [7]

$$
\partial_{t} F+[b, F]=0, \quad \partial_{t} W+[b, W]=[\psi, F] / d_{e}^{2},
$$

with $W=b-d_{e}^{2} \nabla^{2} b$. In Eqs. (1) and (2) $t$ is normalized with the Alfvén and the whistler time, respectively. Equations (1) and (2) are Hamiltonian (see Refs. [5,7]) with energy functionals given by $\int d x d y\left[|\nabla \psi|^{2}+\right.$ 
$\left.|\nabla \varphi|^{2}+d_{e}^{2}\left|\nabla^{2} \psi\right|^{2}+\varrho_{s}^{2}\left|\nabla^{2} \varphi\right|^{2}\right] / 2$ and $\int d x d y\left[|\nabla \psi|^{2}+\right.$ $\left.|b|^{2}+d_{e}^{2}\left|\nabla^{2} \psi\right|^{2}+d_{e}^{2}|\nabla b|^{2}\right] / 2$. In Eq. (2) the generalized flux function $F$ is a Lagrangian invariant advected by the velocity field obtained from the "stream function" $b$. For scale lengths much larger than $d_{e}$, the conservation of $F$ reduces to the conservation of the magnetic flux function $\psi$. As shown in Ref. [5], Eqs. (1) can be rewritten in terms of two generalized flux functions $G_{ \pm}=F \pm d_{e} \varrho_{s} U$ as $\partial_{t} G_{ \pm}+\left[\varphi_{ \pm}, G_{ \pm}\right]=0$, with stream functions $\varphi_{ \pm}=\varphi \pm$ $\left(\varrho_{s} / d_{e}\right) \psi$. For $\varrho_{s} \rightarrow 0$ (cold electrons) the Lagrangian invariants $G_{ \pm}$become degenerate and reduce to $F$. In this limit $F$ is the only Lagrangian invariant and the algebraic structures of Eqs. (1) and (2), i.e., their generalized linking conditions, coincide (with $\varphi$ substituted for $b$ ) while their energy functionals remain different, as the time derivative in the second of Eqs. (1) involves the fluid vorticity $\nabla^{2} \varphi$ only. The systems (1) and (2) provide two convenient mathematical frameworks in order to study the nonlinear evolution of Hamiltonian reconnection. In Ref. [3] the long term nonlinear development of the reconnection instability in the MHD frequency regime was investigated in a sheared magnetic equilibrium configuration with a null line. Only the nondegenerate case $\rho_{s} \neq 0$ was considered. Taking $\varrho_{s} \sim d_{e}$ and a configuration where a single mode can be linearly unstable, it was shown that the plasma evolves towards a "macroscopically stationary" state with a saturated magnetic island. In this process the advection of the Lagrangian invariants $G_{ \pm}$is laminar (i.e., the stream functions $\varphi_{ \pm}$ remain ordered) and $G_{ \pm}$become spatially mixed (see Fig. 3 of Ref. [3]). This process produces a regular filamentary structure of current and vorticity layers, with increasingly small spatial scales, that eventually fill the island. In the absence of dissipation, energy conservation is ensured by the transfer of magnetic energy $\propto|\nabla \psi|^{2}$ to electron kinetic and internal energy $\propto\left[d_{e}^{2}\left|\nabla^{2} \psi\right|^{2}+\right.$ $\left.\varrho_{s}^{2}\left|\nabla^{2} \varphi\right|^{2}\right]$ at increasingly small spatial scales. In the present Letter we show that in the cold electron MHD and EMHD regimes, the saturation of the reconnection instability due to the mixing process leads to a turbulent current layer pattern. This different behavior occurs because in these regimes the Lagrangian advection is subject to a secondary K-H instability which destroys the regular development of the current pattern. In the MHD regime the K-H instability appears only when $\varrho_{s} / d_{e}$ is decreased, while it is always present in the adopted EMHD model where electron temperature effects are neglected.

In order to investigate the onset of a secondary instability during the nonlinear phase of the reconnection process, we have integrated both Eqs. (1) and (2) numerically, using a new version of the 2D fluid code adopted in Ref. [3]. This code advances the cell averaged values of $F$ and $U$ in time (or of $F$ and $W$ in the EMHD case) using a finite volume technique (i.e., calculating the cell fluxes). A Fourier transform method is then used to reconstruct the grid point values of $F$ and $U$ at the cell corners. Time is advanced using the explicit third order AdamsBashforts scheme. Typical mesh sizes are $N_{x}=2048$ and $N_{y}=512$. By looking at the energy and invariants conservation in different regimes and for different grid and time resolutions, we have found that this algorithm is more accurate and faster than the one used in Ref. [3].

First we consider the MHD regime and initialize Eqs. (1) by imposing perturbations of the form $\left[\psi_{1}(x), \varphi_{1}(x)\right] \sum_{k_{y}} \epsilon_{k_{y}} \exp \left(i k_{y} y+\sigma_{k_{y}}\right)$ (with $\epsilon_{k_{y}}$ and $\sigma_{k_{y}}$ random amplitudes and phases, $\psi_{1}(x)$ and $\varphi_{1}(x)$ random functions of $x$ ) on the static equilibrium configuration $\psi_{0}(x)=-L /\left[2 \cosh ^{2}(x / L)\right]$ in a simulation box with $L_{x}=2 L_{y}=4 \pi L$, taking $d_{e}=3 / 10 L$. In this null line magnetic equilibrium only the lowest wavelength eigenmode is unstable and, as in the cases investigated in Refs. [2-4], has a large value of the stability parameter $\Delta^{\prime}$ corresponding, for the chosen values of $d_{e}$, to a "fast" growing instability. In these numerical runs we have considered values of $\varrho_{s} / d_{e}$ ranging from 0 to 1.5 . In Fig. 1 we show the time evolution of the Lagrangian invariant $F$ in the degenerate case $\varrho_{s}=0$ at $t=90 \times 10$. The magnetic flux function $\psi$, as shown in the first two frames by the black contour lines, displays a smooth
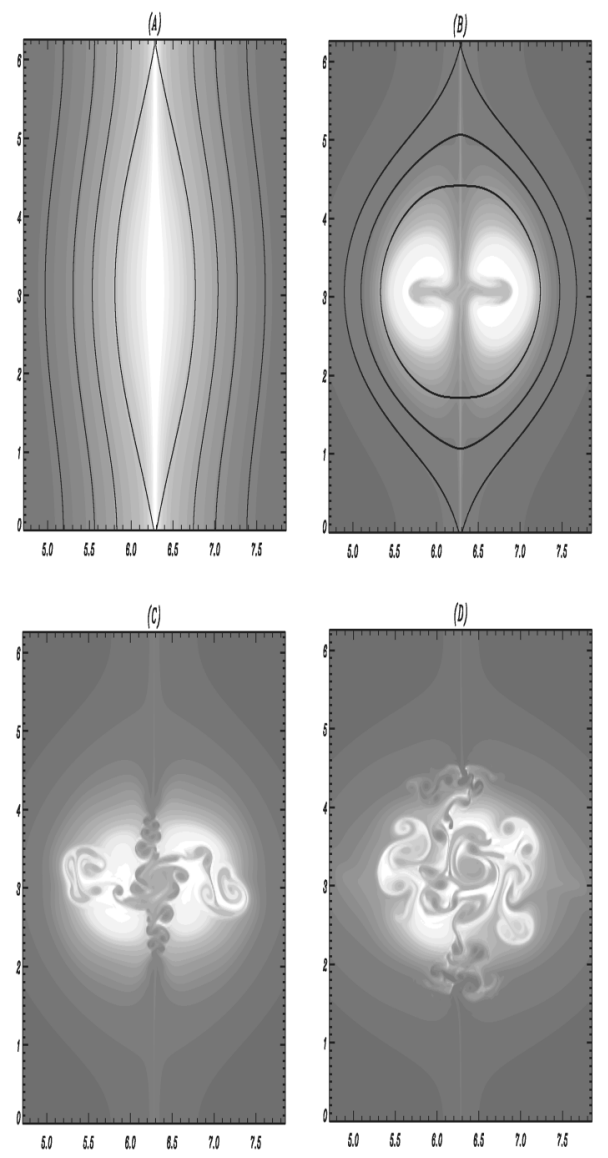

FIG. 1. Shaded isocontours of the Lagrangian invariant $F$ [MHD regime, Eqs. (1)], at $t=90,102,106,111$ for $\varrho_{s}=0$ with superposed, in the upper frames, the isocontours of the magnetic flux function $\psi$. 
structure with a finite size magnetic island and reconnected field lines. On the contrary, the shaded contours of the Lagrangian invariant $F$ (initially straight lines along $y$ ) do not reconnect. In the first phase of the reconnection instability the contours of $F$, which are advected along the hyperbolic cell pattern of the stream function $\varphi$, are pushed towards the $X$ point of $\psi$, leading to the formation of a bar-shaped current layer along the equilibrium null line, frame (a), as first found in the early nonlinear phase in Ref. [4]. At later times, the $F$ contours are stretched in the $y$ direction and carried towards the $O$ point inside the magnetic island, where they encounter the corresponding contours from the adjacent $X$ point. Subsequently, they are advected outwards in the $x$ direction, as shown in frame (b) at $t=102$. At this stage $F$ starts to be affected by the K-H instability that causes a full redistribution of $F$, as shown at $t=$ 106, 111 in frames (c),(d), and of the current density $-\nabla^{2} \psi$. In frame (d) the spatial structure of $F$ is dominated by the twisted filaments of the current density which spread through the central part of the magnetic island. The corresponding evolution of the vorticity $\nabla^{2} \varphi$ is shown in Fig. 2. In frame (d) we see a well developed turbulent distribution of monopolar and dipolar vortices. This turbulent evolution of the nonlinear reconnection
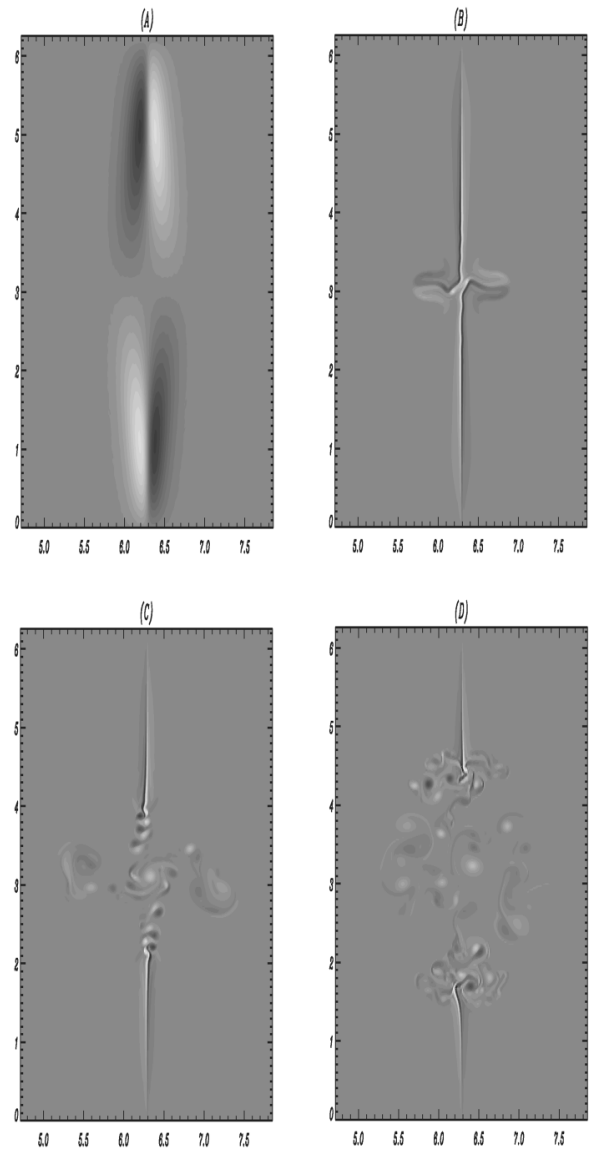

FIG. 2. Shaded isocontours of the fluid vorticity $U=\nabla^{2} \varphi$ for the same parameters and times as in Fig. 1. process persists in the nondegenerate, finite electron temperature case where the two Lagrangian invariants $G_{ \pm}$ determine the generalized linking conditions. However, as the ratio $\varrho_{s} / d_{e}$ is increased, i.e., as the electron temperature effects become more important, the onset of the $\mathrm{K}-\mathrm{H}$ instability occurs later during the island growth and its effect on the current layer distribution becomes weaker. For $\varrho_{s} / d_{e} \sim 1$, no sign of a secondary instability is detectable during the time the island takes to saturate its growth, in agreement with the results of Ref. [3], as also shown here by the laminar advection of $G_{+}$for $\varrho_{s} / d_{e}=1.5$ in Fig. 3. In the transitional regime, the advection pattern and the current layer structures exhibit an intermediate behavior, as shown for $\varrho_{s} / d_{e}=0.167$ in Fig. 4. Initially, $G_{ \pm}$are advected in opposite directions with a differential rotation, as described in Ref. [3] for $\varrho_{s} / d_{e}=1$. At later times [frame (b) in Fig. 4], they acquire features characteristic of the evolution of $F$ in the degenerate $\varrho_{s}=0$ case and their advection becomes K-H unstable [frame (c)] leading to an almost turbulent distribution, as shown in frame (d). A linear analysis of the onset of the K-H instability in a set of model 1D equilibria, chosen so as to mimic the local advection pattern at the reconnection layer for different values of $\varrho_{s} / d_{e}$, indicates that a $\mathrm{K}-\mathrm{H}$ instability does occur and that its growth rate is a decreasing function of $\varrho_{s} / d_{e}$. A comparison of the power law behavior of the $x$-averaged $k_{y}$ Fourier spectra (not shown here) of the Lagrangian invariants, of the fluid stream function $\varphi$, and of the magnetic flux function $\psi$ as $\varrho_{s} / d_{e}$ is varied shows that the slope of the spectrum of $\varphi$ is significantly affected by the development of the $\mathrm{K}-\mathrm{H}$ instability while both the spectra of the invariants and, in particular, that of the flux function $\psi$ are almost unchanged. The spectra of the Lagrangian invariants are flatter than that of $\psi$ while that of $\varphi$ has an intermediate slope, becomes steeper as $\varrho_{s} / d_{e}$ is increased, and tends to coincide with that of $\psi$. The energy balance (not shown here) indicates an excellent total energy conservation until the fully developed phase of the K-H instability when turbulent motions

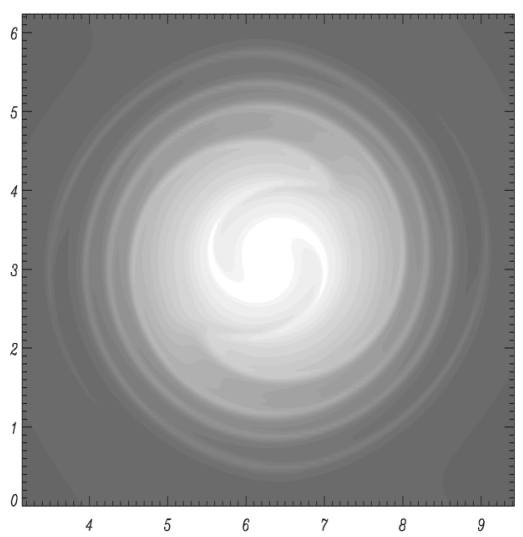

FIG. 3. Shaded isocontours of the Lagrangian invariant $G_{+}$ [MHD regime, Eqs. (1), for $\varrho_{s} / d_{e}=1.5$ ], at $t=80$. 

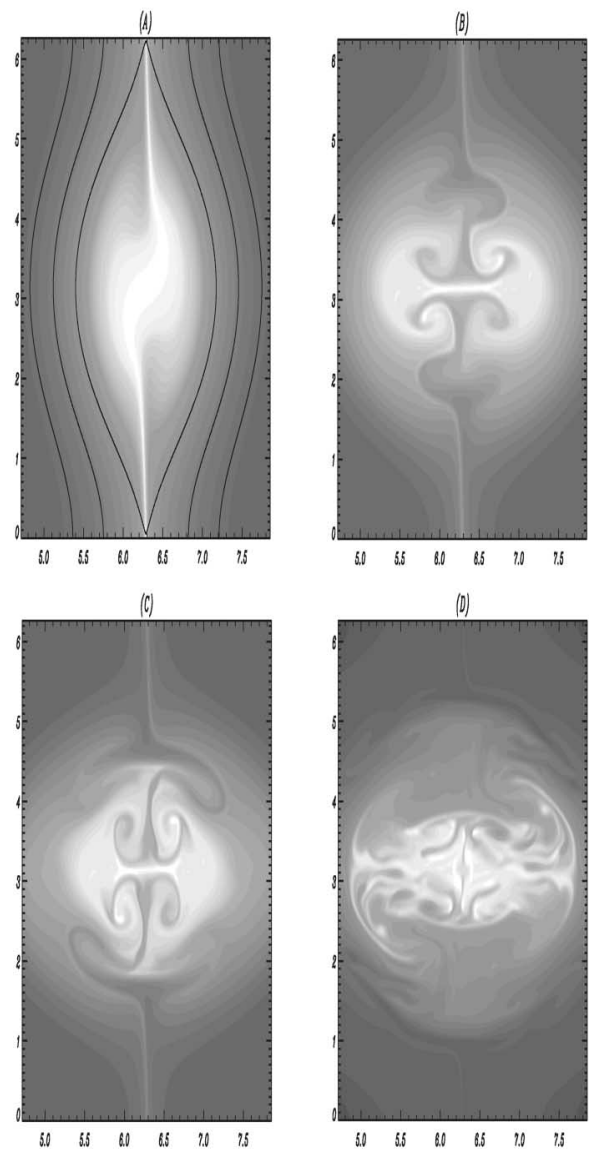

FIG. 4. Shaded isocontours of the Lagrangian invariant $G_{+}$ at $t=65,75,78,93$ for $\varrho_{s} / d_{e}=0.167$ with superposed, in the first frame, the isocontours of the magnetic flux function $\psi$.

inject energy effectively on the dissipative (grid) length scales. This energy dissipation, which is smaller than the magnetic and kinetic energy variations, occurs faster for small values of $\varrho_{s} / d_{e}$. For these values, part of the magnetic energy released by the reconnection instability remains in the form of plasma kinetic energy $\int d x d y|\nabla \varphi|^{2}$ corresponding to the fluid vortices in the magnetic island. In addition, an oscillatory exchange of energy persists between the plasma kinetic energy and the electron kinetic energy $\int d x d y d_{e}^{2}\left|\nabla^{2} \psi\right|^{2}$, corresponding to "breathing oscillations" of the island shape analogous to those described in Ref. [9]. Because of their algebraic structure equal to that of the degenerate MHD case $\varrho_{s}=$ 0 and different Hamiltonian functional, the EMHD Eqs. (2) allow us to verify the relationship between the onset of the secondary instability and the form of the conserved generalized linking conditions. The results of the numerical integration of Eqs. (2) presented in Fig. 5 for the same equilibrium configuration and initial conditions as in Fig. 1 (with $b_{0}=$ const and $b_{1}$ substituted for $\varphi_{1}$ ) show that the onset and the development of the $\mathrm{K}-\mathrm{H}$ instability in the EMHD regime and the evolution of the Lagrangian invariant $F$, correspond fully to those
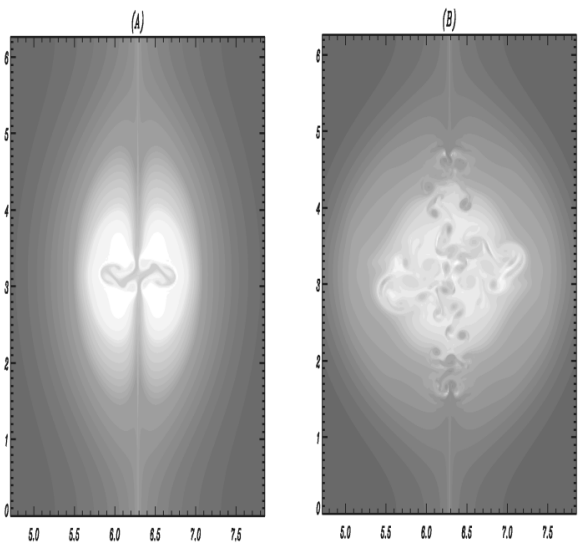

FIG. 5. Shaded isocontours of the Lagrangian invariant $F$ [EMHD regime, Eqs. (2)], at $t=132,141$.

shown in Figs. 1 and 2 and is accompanied by formation of turbulent current filaments and of long lasting electron vortices both in $F$ and in $W$ (not shown).

In conclusion, we have shown that in the fully nonlinear phase of (fluid) Hamiltonian magnetic reconnection the K-H instability appears, even in the absence of an equilibrium velocity shear, as a secondary instability that makes the advection of the conserved Lagrangian invariants turbulent. These new results indicate that the mixing process of the Lagrangian invariants identified in [3] in the nondegenerate MHD case represents a natural feature of 2D Hamiltonian reconnection but that it can develop in different features depending on whether secondary instabilities, such as the $\mathrm{K}-\mathrm{H}$ instability, are excited. Thus, although in all the regimes examined the current layers eventually cover a large portion of the island domain, their spatial structure and the magnetic energy redistribution can develop very differently depending on the role played by the electron thermal effects which change the form of the conserved generalized linking conditions.

Part of this work was supported by the INFM Parallel Computing Initiative and by the Italian Ministry for University and Scientific Research PRIN funds.

[1] D. Biskamp, Phys. Plasmas 4, 1964 (1997).

[2] E. Cafaro et al., Phys. Rev. Lett. 80, 4430 (1998).

[3] D. Grasso et al., Phys. Rev. Lett. 86, 5051 (2001).

[4] M. Ottaviani et al., Phys. Rev. Lett. 71, 3802 (1993).

[5] B. N. Kuvshinov et al., Phys. Lett. A 191, 296 (1994); B. N. Kuvshinov et al., J. Plasma Phys. 59, 727 (1998).

[6] A. S. Kingsep et al., in Reviews of Plasma Physics, edited by (Consultants Bureau, New York, 1990), Vol. 16, p. 243.

[7] S.V. Bulanov et al., Phys. Fluids B 4, 2499 (1992); K. Avinash et al., Phys. Plasmas 5, 2849 (1998); V.P. Ruban, Phys. Rev. E 65, 047401 (2002).

[8] D. Biskamp, Phys. Plasmas 6, 751 (1999).

[9] J. Bergmans et al., Phys. Rev. Lett. 87, 195002 (2001). 